\title{
Application of ground penetrating radar in concrete defect detection of sluice floor
}

\author{
Ye wu ${ }^{1}$, Wei Yong-xia ${ }^{2}$, Xu Ming-xian ${ }^{3}$, Ye Lin-xiang ${ }^{4}$ \\ ${ }^{1}$ Zhejiang Haichuan Survey Co., Ltd, Hangzhou 310000, China \\ ${ }^{2}$ Zhejiang Zhengyuan Hydropower Survey and Design Institute Co., Ltd, Hangzhou 310051, China \\ ${ }^{3}$ Zhejiang Design Institute of Water Conservancy and Hydroelectric Power, Hangzhou Zhejiang 310002, China \\ ${ }^{4}$ Kunming Engineering Survey Company, Kunming , 650100, China
}

\begin{abstract}
The defect investigation of sluice floor is an important measure to ensure the safe operation of the sluice. due to the traditional drilling methods will cause damage to the sluice floor, and the appearance inspection and compressive strength test can not evaluate the internal defects of reinforced concrete in the sluice floor. In this paper, we apply the ground penetrating radar(GPR) to the concrete quality inspection of a sluice gate in a seawall. And we describe the layout, detection principle and data processing flow of GPR. Then we simulate the propagation characteristics of radar electromagnetic wave in the presence of twodimensional electrical anomaly bodies by finite difference method. Through analyzing the amplitude, frequency and event characteristics of reflection wave shown in the gray map of GPR profiles, we determine the dividing line between concrete and muddy silty clay, finding out the density of steel in concrete, framing the location of construction joints and suspected water-filled gaps.
\end{abstract}

\section{Introduction}

A project is located in Jiaxing City Zhejiang Province. It is one of the key projects for comprehensive management of Taihu Lake Basin, which focuses on flood control and waterlogging removal, and also takes into account irrigation and water supply, shipping and water environment improvement. As one of the control systems of the project, the concrete quality of the bottom slab of the sluice chamber and adjacent apron and stilling basin has an important impact on the safe operation and drainage effect of the sluice. The common concrete detection methods mainly include appearance inspection, compressive strength test and radar detection. The first two methods can only qualitatively evaluate the quality of concrete, but can not detect its internal structure. Ground penetrating radar (GPR) is a geophysical method that uses high-frequency wireless electromagnetic wave to determine the distribution law of materials inside the medium. It has the characteristics of high resolution, high efficiency and no damage to the medium which widely used in the fields of engineering [1], environment ${ }^{[2]}$, geological survey ${ }^{[3]}$ and archaeology ${ }^{[4]}$.

In this paper, the GPR is used to detect the internal quality of the sluice floor concrete. The radar wave detection mechanism and the corresponding data processing method are introduced. The radar profile can better reflect the distribution of concrete construction joints and reinforcement. Through the analysis of radar wave amplitude and frequency, the division between concrete and underlying muddy silty clay layer is drawn boundary line.

\section{The layout of GPR measuring line}

The engineering grade of the sluice is grade I, the standard of dampproof is once in 100 years, the designed drainage flow of the project is $664 \mathrm{~m}^{3} / \mathrm{s}$, and the design thickness of the concrete for the bottom plate of the sluice chamber is $1 \mathrm{~m}$. The main media in the survey area are air, water, floor concrete and underlying soil layer. The physical properties of concrete and soil are quite different. The former has the characteristics of high resistance and low dielectric, while the latter has relatively low resistivity. Its relative permittivity is less than 10 in dry environment and 30 in wet environment. There are obvious physical properties differences between the sluice floor concrete and the underlying soil layer, which has the geophysical premise of GPR method.

A total of thirteen meshed GPR survey lines (figure 1) are arranged in the survey area with a total length of $514 \mathrm{~m}$. Among them, there are 4 longitudinal lines with No.001No.004 and nine horizontal lines with No.005- No.013.

\footnotetext{
*Corresponding author: haichuankancha@126.com
} 


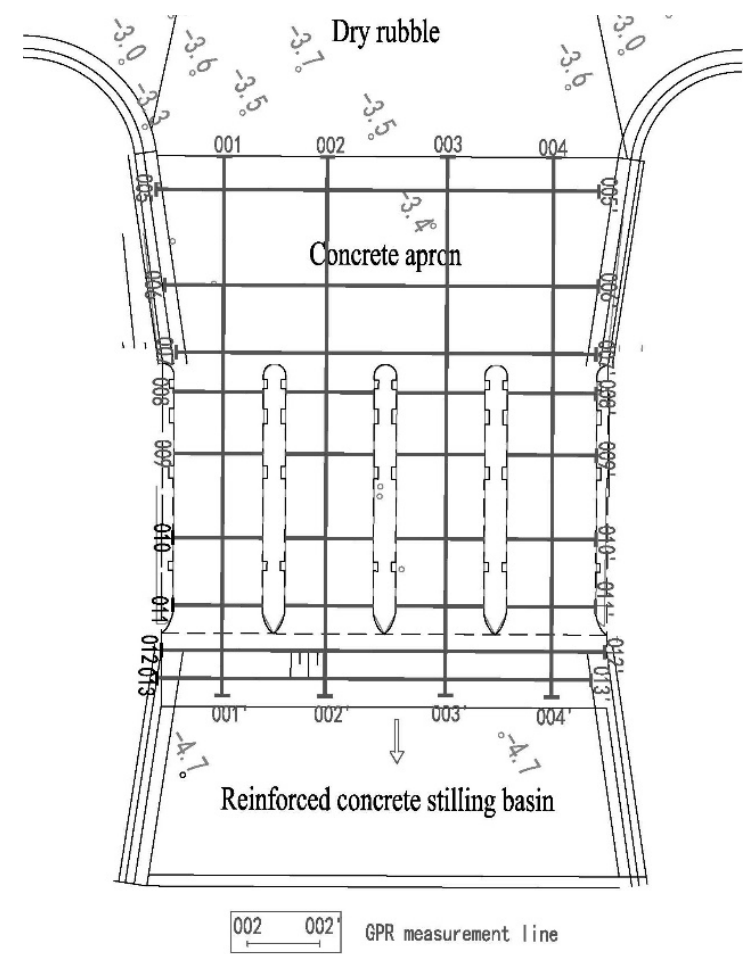

Figure 1. GPR survey line layout in gate site area

\section{Detection principle of GPR}

GPR is a non-destructive testing technology which has the advantages of loose working conditions and high detection accuracy. In the form of broad-band short pulse, radar wave is sent into the ground by transmitting antenna and then return to the ground after reflected by underground stratum or target, and received by receiving antenna. The propagation process of radar wave satisfies the wave equation. In order to show the detection mechanism intuitively, the three-layer inclined medium model as shown in figure 2 is calculated by FDTD. The first layer is air layer with thickness of $1 \mathrm{~m}$, and its conductivity $\sigma_{1}=0 \mathrm{~S} / \mathrm{m}$ and relative permittivity $\varepsilon_{1}=1$.The conductivity of second layer $\sigma_{2}=0.001 \mathrm{~S} / \mathrm{m}$ and the

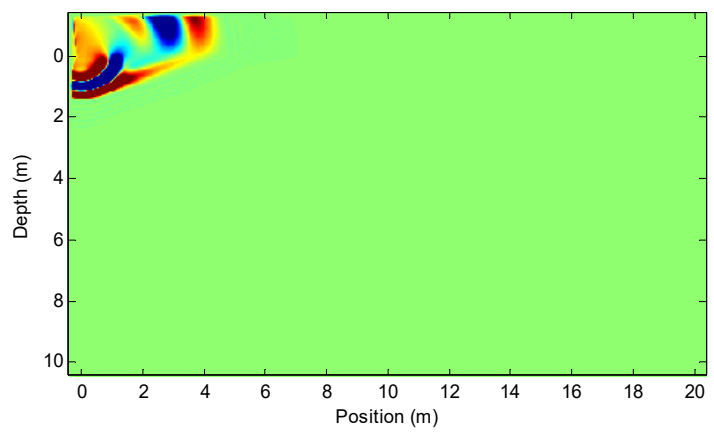

(a) $\mathrm{T}=16 \mathrm{~ns}$ corresponding relative permittivity $\varepsilon_{2}=9$, in this layer, there are two abnormal bodies with the size of $3 \mathrm{~m} \times 1 \mathrm{~m}$ and $6 \mathrm{~m} \times 1.5 \mathrm{~m}$ respectively, the distance from the second abnormal body to the left boundary is $5 \mathrm{~m}$, and the relative permittivity $\varepsilon_{A}=\varepsilon_{B}=16$. The conductivity and relative permittivity of the third layer are $\sigma_{3}=0.005 \mathrm{~S} / \mathrm{m}$ and $\varepsilon_{3}=25$ respectively, and the dip angle of inclined plane is $11.3^{\circ}$. The calculation area is $20 \mathrm{~m} \times 11 \mathrm{~m}, 200 \times 110$ grids are used, the grid spacing is $0.1 \mathrm{~m}$, and the main frequency of radar wave is $283 \mathrm{MHz}$.

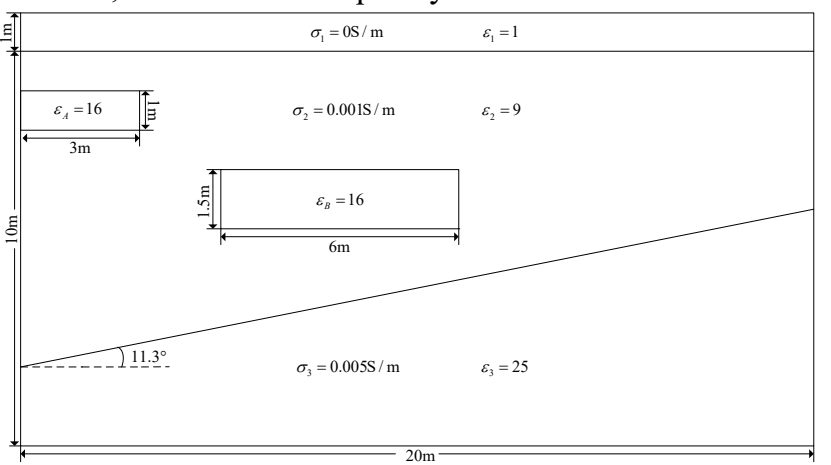

Figure 2. Two dimensional geological model for simulating radar wave propagation

Figure 3 shows the wave field snapshot of the horizontal component of the electric field of the radar wave calculated by the finite difference time domain(FDTD) method shown in figure 2 at different times. As shown in figure 3, the radar wave first diffuses outward in a circular shape(figure $3 \mathrm{a}$ ), after encountering anomaly body A, upward reflection wave appears(figure $3 b)$. Part of the electromagnetic wave continues to propagate downward, and the reflected wave appears again after encountering abnormal body B (figure 3c), at the time of $\mathrm{T}=88 \mathrm{~ns}$, the front of the wave reaches the inclined plane, and the waveform begins to compress(figure $3 \mathrm{~d}$ ), and then the reflected wave on the plane appears(figure 3e). The curvature of the front of the reflected wave at this interface is small. Finally, the radar wave is divided into obvious up and down traveling waves(figure 3f).

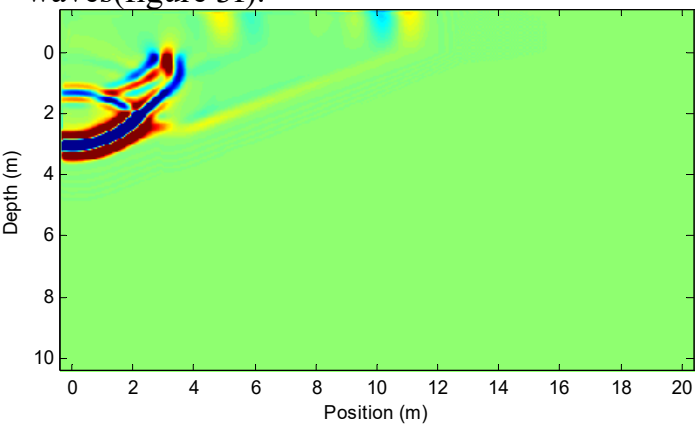

(b) $\mathrm{T}=40 \mathrm{~ns}$ 


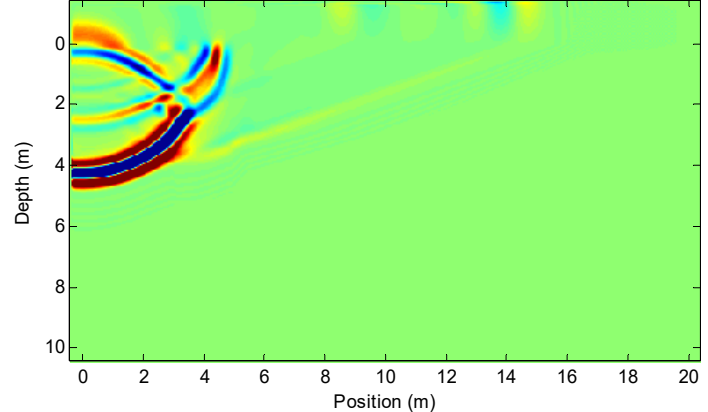

(c) $\mathrm{T}=52 \mathrm{~ns}$

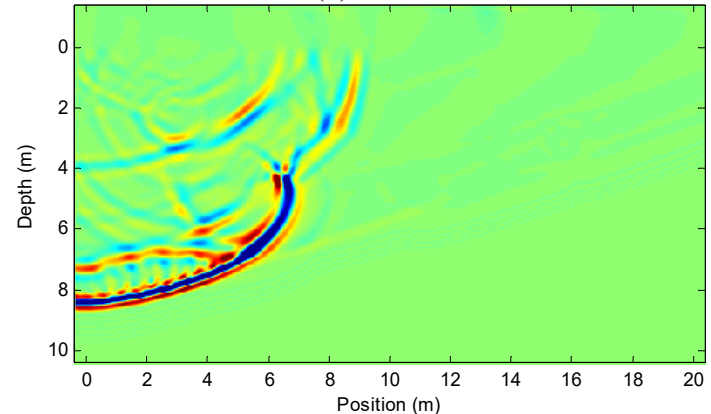

(e) $\mathrm{T}=96 \mathrm{~ns}$

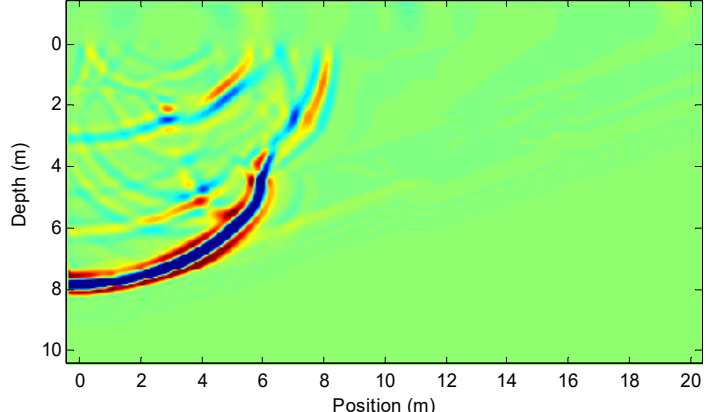

(d) $\mathrm{T}=88 \mathrm{~ns}$

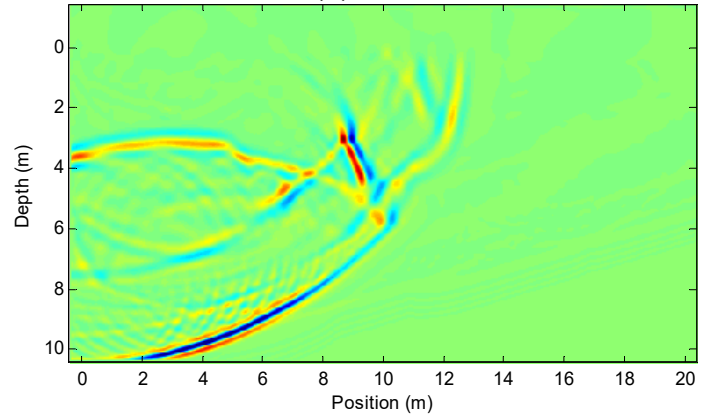

(f) $\mathrm{T}=132 \mathrm{~ns}$

Figure 3. Radar wave field snapshot at different times

\section{Processing and interpretation of GPR data}

$500 \mathrm{MHz}$ shielded antenna is used in this radar detection. The effective detection depth can reach $6 \mathrm{~m}$ on the concrete with high resistance and low dielectric. The vertical resolution can reach $0.1 \mathrm{~m}$. The transverse resolution is related to the detection depth, and can reach $0.03-0.11 \mathrm{~m}$ within $2 \mathrm{~m}$. Radar data processing mainly includes subtract-DC-shift, static correction, energy decay gain, offset, band-pass filtering and moving average.

The main purpose of this radar detection is to find out whether there are obvious defects in the concrete of the station sluice floor and the corresponding thickness of the bottom plate. Therefore, this paper only analyzes the typical radar profiles with obvious anomalies. The radar profile is represented by gray-scale image, the principle is to describe the area with weak amplitude of reflected signal as gray area, the wave trough phase of reflection interface as black, and the peak phase as white.

The more black or white, the stronger the amplitude. The signal frequency is expressed by the thickness of black and white squares, the greater the thickness, the lower the frequency.

\subsection{GPR results interpretation of measuring line 2-2'}

The survey line 2-2' passes through the apron section, gate chamber section and stilling basin section, which is a longitudinal survey line. Figure 5 is the gray map of GPR profile of survey line 2-2'. As shown in figure 5, there are many single hyperbolas near the depth of about $0.2 \mathrm{~m}$. The corresponding position of the hyperbola top is the top of the cylindrical reinforcement in the concrete. The spacing of the hyperbola represents the density of the reinforcement. It can be seen from the figure that the spacing between the apron and the gate bottom plate reinforcement is about $0.25 \mathrm{~m}$. Near the horizontal distance of $18.4 \mathrm{~m}$, there is a slightly large range of hyperbolic anomaly with large amplitude. The projection of the top of hyperbola on the ground corresponds to the construction joint between the surface apron and the sluice chamber. There is a reflection wave with continuous phase axis and strong amplitude near the depth of about $1 \mathrm{~m}$, which can be inferred as the interface between concrete and underlying soil layer (marked by red line in figure 4 ).

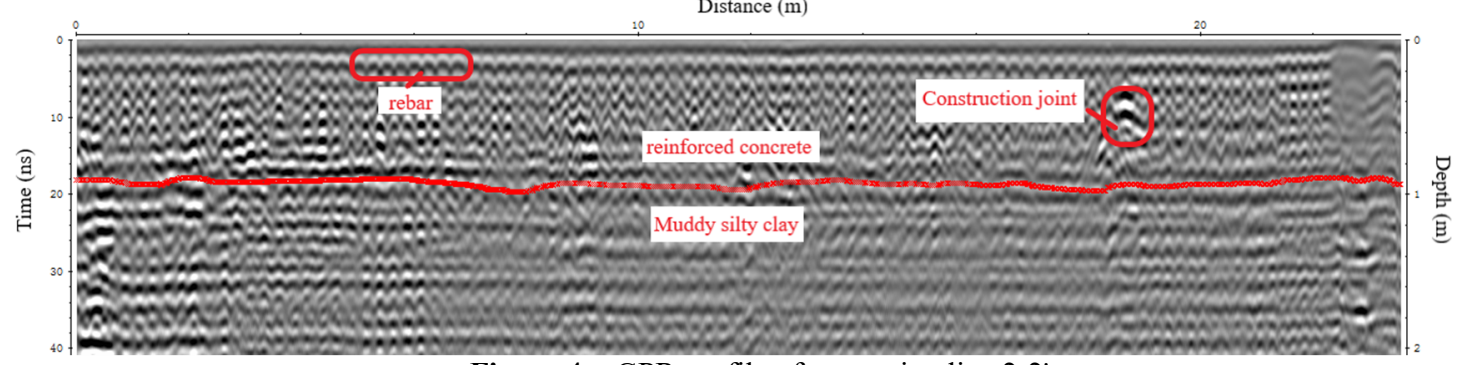

Figure 4. GPR profile of measuring line 2-2' 


\subsection{GPR results interpretation of measuring line 4-4'}

Figure 5 shows the gray-scale map of GPR profile of survey line 4-4 '. As shown in figure 5, the abnormal law of reflected wave in the radar profile is similar to that of section 2-2 ', but there is a banded anomaly in the horizontal distance of $1-15 \mathrm{~m}$ with the time domain of 10 20ns. The abnormal of reflection wave has good continuity event, strong amplitude and low main frequency. This anomaly is inferred to be construction joint and there is a small amount of water filling in the gap.

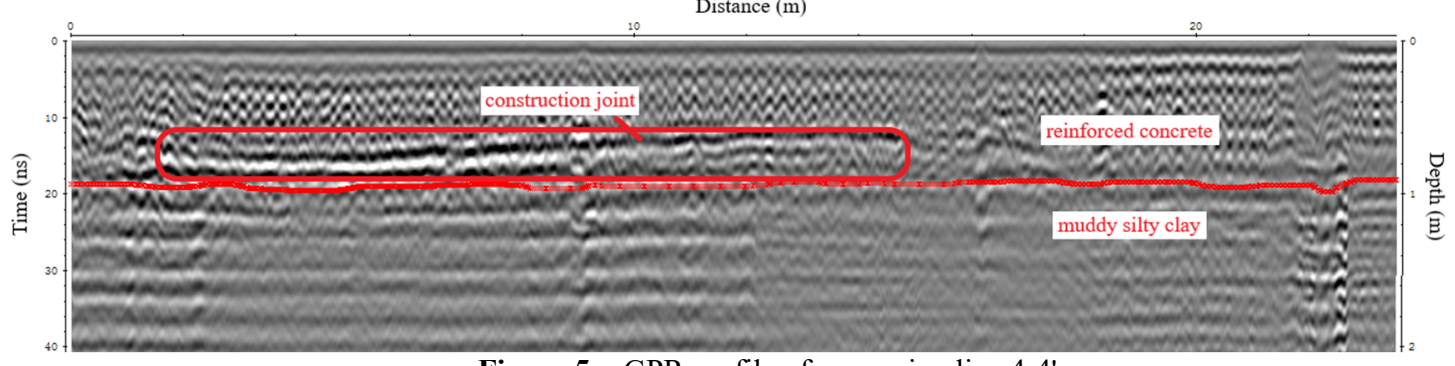

Figure 5. GPR profile of measuring line 4-4'

We compared the anomalies shown in figure 4 and figure 5 with the design data which proved to be the horizontal and longitudinal construction joints, and then verified the effectiveness of GPR in the detection of reinforced concrete defects in sluice floor.

\section{Conclusions}

In this paper, the internal structure of concrete in the bottom slab of a sluice and its adjacent apron and stilling basin is detected by GPR. The results show that the method can accurately reflect the thickness of concrete floor, weak zone and the distribution characteristics of internal reinforcement. At the depth of about $1 \mathrm{~m}$, the reflected wave has continuous event and strong amplitude, which corresponds to the boundary between concrete and underlying soil layer. On the radar section, the reinforcement is one by one hyperbola with similar shape. The position with the strongest amplitude at the top of the hyperbola is the buried depth of the reinforcement, and the spacing of the hyperbola corresponds to the density of the reinforcement. When there is a water filled crack in the concrete, the event of the reflected wave shows white banded shape on the gray-scale image of the radar section. The simulated radar wave field snapshot at different time in this paper is helpful to understand the detection mechanism of electromagnetic wave quickly. And the working method and research results of GPR discussed in this paper can provide useful reference for the rapid evaluation of the internal quality of sluice floor.

\section{References}

1. Liu, Z. H Liu, M. M Zhou, D (2019) Recognition method of typical anomalies in karst tunnel construction based on ground penetrating radar attribute analysis[J]. Rock and Soil Mechanics, 40(8): $1-9$.

2. Guo, C. G Guo, Y. F Zhu, M. L Zhang, Q. W (2020) Hydrogeological investigation of the underground river of karst tunnel in carbonate rock of red bed[J]. Modern Tunnelling Technology, 57(1): 58-64.
3. Ma, C. H Chen, J (2019) Application of geological radar technology to investigations of hazardous debris flow: An example from Nan'an, Fangshan District, Beijing[J]. Geology and Exploration, 55(4): 10661072.

4. Qin, T Zhao, Y. H Lin, G. C, Hu, S. F An, C Geng, D. X Rao, C F (2018) The application of GPR to underwater archaeological investigation of Shanglinhu Yue kiln relics. Geophysical and Geochemical Exploration, 42(3): 624-630. 